\title{
Acute febrile neutrophilic dermatosis in a patient with Crohn's disease: case report and review of the literature
}

\author{
Pavel Skok ${ }^{1 凶}$, Kristijan Skok ${ }^{2}$
}

\begin{abstract}
Crohn's disease is a chronic inflammatory bowel disease. The disease is characterized by acute exacerbations with diarrhea, abdominal pain, fever, anorexia, intestinal bleeding, and weight loss. Immune-mediated diseases that are frequently associated with Crohn's disease include arthritis, ankylosing spondylitis, sacroiliitis, episcleritis, uveitis, and skin lesions, such as erythema nodosum and pyoderma gangrenosum. The authors present the case of a 22 -year-old female patient that was admitted to their hospital due to diarrhea, fever, arthralgias, and diffuse erythematous papules and plaques with vesicles and pustules affecting the patient's face, lips, arms, trunk, and legs. Six months prior to onset, the patient was diagnosed with terminal ileitis and Crohn's disease of the sigmoid colon. Treatment with mesalazine and budesonide had been introduced. In the diagnostic procedure, a skin biopsy was taken from the patient. Histology confirmed dense infiltration of neutrophilic polymorphonuclear leukocytes, or Sweet's syndrome. This condition is a rare manifestation of chronic inflammatory bowel diseases such as Crohn's disease and ulcerative colitis. This syndrome is thought to be a hypersensitivity reaction and may be associated with various inflammatory, infectious, or neoplastic diseases. The patient was treated with high doses of methylprednisolone (6o mg IV), which resulted in rapid improvement of Crohn's disease and skin lesions.
\end{abstract}

Keywords: Crohn's disease, immune-mediated diseases, acute neutrophilic dermatosis, complications, Sweet's syndrome

Received: 25 January 2018 | Returned for modification: 27 May 2018 | Accepted: 11 June 2018

\section{Introduction}

Crohn's disease (CD) is a chronic inflammatory bowel disease (IBD); in most cases, it affects the distal ileum and various parts of the colon. It was first described by Burrill Bernard Crohn and two colleagues, Leon Ginzburg and Gordon Oppenheimer, in 1932 and named regional enteritis or terminal ileitis (1). Since 1960 it has been known that inflammatory changes of the bowel mucosa can be found anywhere from the mouth to perianal region (2). In North America, the annual incidence of CD is 3.1 to 20.2 per 100,000 with a prevalence of 201 per 100,000. In western Europe the incidence is 10 per 100,000 and the prevalence 100 per 100,000. In Slovenia, the incidence is 5 per 100,000; unfortunately, there are no precise data on the prevalence $(3,4)$. Epidemiological, genetic, and immunological data show that $\mathrm{CD}$ is a heterogeneous disorder with a multifactorial etiology in which genetics and environmental factors trigger the disease. The peak incidence of the disease is between the second and fourth decade of life. Both sexes are equally affected. Injury of the intestinal wall is the consequence of a disturbed immune response to intestinal bacterial infection. Inflammation often spreads to all layers of the intestinal wall, as well as the peritoneum and nearby lymph nodes. The disease is characterized by acute exacerbations with diarrhea, abdominal pain, intestinal bleeding, fever, anorexia, and weight loss. Among frequent complications are strictures or stenosis of the bowel, fistulas, and abscesses.

Arthritis, episcleritis, aphthous stomatitis, ankylosing spondylitis, sacroiliitis, uveitis, sclerotic cholangitis, and skin lesions, which include erythema nodosum and pyoderma gangrenosum, are also frequent manifestations of these associated immune-mediated diseases (5).

The authors present the case of a female patient, that, six months after chronic IBD of the ileum and sigmoid colon had been discovered, developed a rare manifestation of exacerbation of $\mathrm{CD}$ in the form of acute febrile neutrophilic dermatosis, also known as Sweet's syndrome.

\section{Case report}

A 22-year-old female patient was admitted to our hospital from a regional facility due to fever, diarrhea, arthralgias, and disseminated erythematous papules and plaques with vesicles and pustules on the face, lips, arms, trunk, and legs (Figs. 1, 2). Her oral mucosa and eyes were not involved. The skin changes had been evolving over the last 4 days prior to admission to our ward. During treatment at the regional hospital, urinary infection was confirmed and treatment with ciprofloxacin and metronidazole was introduced. Six months prior to this onset, terminal ileitis and CD of the sigmoid colon had been diagnosed with endoscopy and histology. Treatment with mesalazine and budesonide had been introduced. On admission to our department, the patient was eupneic with blood pressure $120 / 80 \mathrm{mmHg}$, pulse $110 / \mathrm{min}$, and febrile $\left(39.5^{\circ} \mathrm{C}\right)$. Laboratory findings confirmed anemia with hemoglobin values $85 \mathrm{~g} / 1$ (normal 120-150 g/l) and leukocytosis $14.3 \times 109 / 1$ (normal white blood cell count 4.0-10 × 109/1). C-reactive protein (CRP) was elevated to $110 \mathrm{mg} / 1$ (normal o-6 mg/l). The serum iron values were moderately reduced, $7.4 \mu \mathrm{mol} / 1$ (normal 10.7-28.6 $\mu \mathrm{mol} / \mathrm{l}$ ), and the ferritin values elevated, $518 \mu \mathrm{g} / \mathrm{l}$ (normal 20-200 $\mu \mathrm{g} / \mathrm{l}$ ). Serum protein level was decreased to $60 \mathrm{~g} / \mathrm{l}$ (normal 65-80 g/l), but serum albumin was still within normal range. Blood sugar, serum levels of transaminases, urea, creatinine, bilirubin, electrolytes, and lipids, including triglycerides, were normal.

The patient was treated with intravenous infusion of fluids, 
analgesics, antibiotics, transfusion (700 ml), and iron in IV form (1,00o mg). While treatment was initiated, diagnostic procedures were performed to elucidate the etiology of the skin eruptions. Various viral and other infections were excluded. After consultation with a dermatologist, a skin biopsy was performed. Histology confirmed a dense infiltration of neutrophilic polymorphonuclear leukocytes, or Sweet's syndrome (Fig. 3). The introduction of methylprednisolone (6o mg IV) led to rapid improvement of $\mathrm{CD}$ and skin lesions. In the following days, the patient's condition improved rapidly, and leukocytosis and CRP levels decreased within a few days. The patient was discharged 14 days later with minimal sequalae on skin lesions. In the timespan of 2 years after the discharge, due to the unfavorable course of $\mathrm{CD}$, we had to introduce biological drugs, TNF-alfa inhibitors (infliximab), and immunomodulators (azathioprine), which are still being used. In the meantime, the patient gave birth to a healthy daughter.

\section{Discussion}

CD is a chronic IBD caused by disturbed immune response to commensal intestinal microflora in genetically susceptible individuals (2-4). It can affect any segment of the digestive tract and has a relapsing-remitting course. The prevalence and incidence of CD has increased significantly over recent decades in developed parts of the world. The course of the disease is often unpredictable, with the involvement of various organs and complications. Skin is one of the most commonly involved sites. Skin lesions are usually classified as reactive skin findings, specific skin lesions, and nutritional skin changes $(5,6)$.

Pyoderma gangrenosum and acute febrile neutrophilic dermatoses, the latter also known under the eponym of Sweet's syndrome, are severe dermatoses of unknown origin often associated with IBD (6). The clinical picture was first described by Robert Douglas Sweet as acute febrile neutrophilic dermatosis in 1964 (7). In the original paper he described the key features of this condition, also called Gomm-Button disease, that he had identified in eight women from 1949 to 1964 . Since then, numerous papers have been published in international literature on this syndrome in association with various conditions (8-10). The pathogenesis of this condition is probably multifactorial and remains unexplained. It is safe to assume that cytokines probably play an important role in its pathogenesis. This syndrome can present itself in various clinical settings: classical or idiopathic Sweet's syndrome, malignancy-associated Sweet's syndrome, and drug-induced Sweet's syndrome $(8,11)$. What is more, a special form known as "histiocytoid Sweet's syndrome" has recently been described. In this condition, the dermal infiltrate is composed of mononuclear cells with a histiocytic appearance that represent immature myeloid cells (12). The classical syndrome is usually diagnosed in women at the age of 30 to 50 years. It is often preceded by an infection and may be associated with IBD and pregnancy. Malignancy-associated Sweet's syndrome can present as a paraneoplastic syndrome in cancer patients or in patients that have not yet been diagnosed with cancer. Drug-induced Sweet's syndrome has been most frequently associated with the use of granulocyte-colony stimulating factor (G-CSF, or filgrastim), but also with other drugs. The first description of the drug-induced syndrome was associated with trimethoprimsulfamethoxazole $(11,13)$.

In our patient, classical Sweet's syndrome was diagnosed, probably due to the exacerbation of the underlying disease. Urinary tract infection and antibiotic treatment is the less likely cause
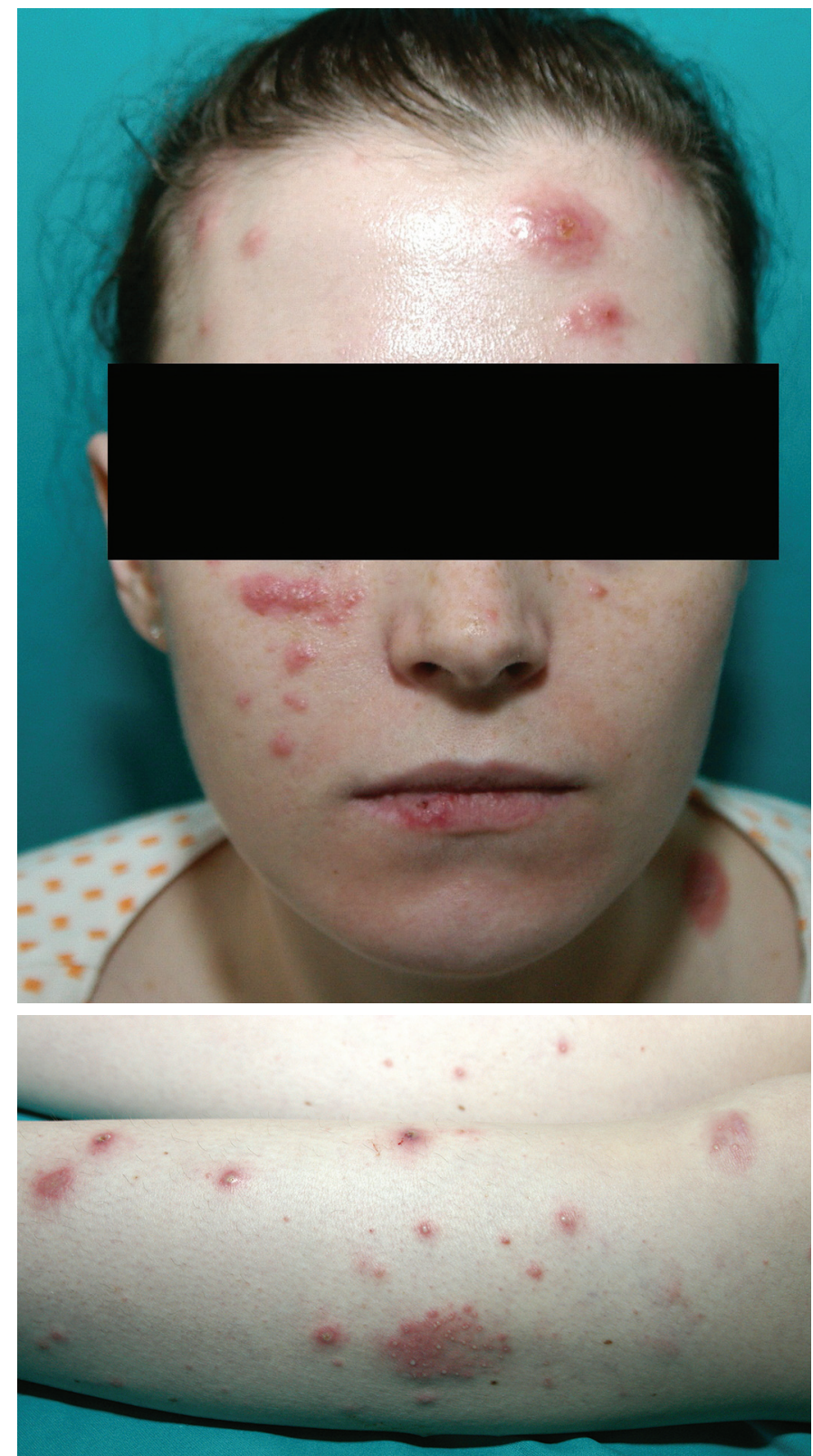

Figures 1, 2 | Disseminated erythematous papules and plaques with vesicles and pustules on the face, lips, and legs.

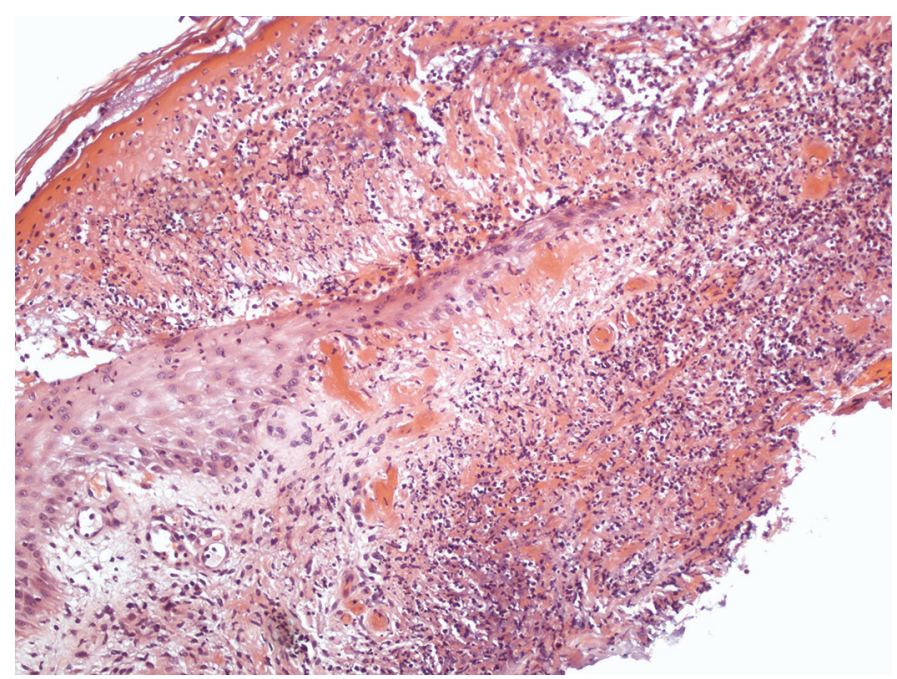

Figure 3 | Punch biopsy of a skin lesion: dense infiltration of neutrophilic polymorphonuclear leukocytes in the dermis.

because our patient had already been treated in the past with metronidazole and ciprofloxacin due to CD exacerbations and 
infections. The features of this syndrome present with a typical clinical picture of fever, leukocytosis, and tender erythematous skin lesions, such as papules, nodules, and plaques disseminated on the face, neck, chest, and limbs. With histology, a diffuse infiltrate predominantly consisting of mature neutrophils typically located in the upper dermis is diagnosed $(6,7)$. The clinical picture and symptoms typically subside in a few days after initiation of systemic corticosteroid therapy. In our female patient, after the introduction of methylprednisolone in IV form, significant improvement in the clinical picture and skin changes was achieved. Even the problems of the affected large intestine were alleviated in the following weeks because steroids are one of the key drugs in IBD (3). During the follow-up period, due to the unfavorable course of CD, we introduced biological drugs, TNF-alfa inhibitors (infliximab), and immunomodulators (azathioprine), which are still being used.

According to the literature, in patients with a refractory form of the syndrome, treatment with potassium iodide, colchicine, indomethacin, clofazimine, cyclosporine, and dapsone has also been

\section{References}

1. Crohn BB, Ginzburg L, Oppenheimer G. Regional ileitis. A pathological and clinical entity. JAMA. 1932:1323-9.

2. Khor B, Gardet A, Xavier RRJ. Genetics and pathogenesis of inflammatory bowel disease. Nature. 2011;474:307-17.

3. Zupančič K, Skok K, Repnik K, Weersma RK, Potočnik U, Skok P. Multi-locus genetic risk score predicts risk for Crohn's disease in Slovenian population. World J Gastroenterol. 2016;22:3777-84.

4. Gajendran M, Loganathan P, Catinella AP, Hashash JG. A comprehensive review and update on Crohn's disease. Disease-a-Month. 2018;64:20-57.

5. Eames T, Landthaler M, Karrer S. Crohn's disease: an important differential diagnosis of granulomatous skin diseases. Eur J Dermatol. 19:360-4.

6. Su CG, Judge TA, Lichtenstein GR. Extraintestinal manifestations of inflammatory bowel disease. Gastroenterol Clin North Am. 2002;31:307-27.

7. Sweet RD. An acute febrile neutrophilic dermatosis. Br J Dermatol. 1964;76:34956.

8. Cohen PR, Kurzrock R. Sweet's syndrome revisited: a review of disease concepts. Int J Dermatol. 2003;42:761-78. used successfully (10). Recently, a patient with this syndrome was successfully treated with granulocyte and monocyte adsorption apheresis (14). After treatment or spontaneous remission, depending on the underlying disease, the syndrome usually has a good prognosis. However, even in patients with non-malignant disease, it may reoccur.

\section{Conclusion}

Sweet's syndrome is a rare manifestation of CD. This syndrome has to be considered in the presence of the characteristic clinical picture and skin changes described above. Early diagnosis of this dermatosis is crucial because of the introduction of systemic corticosteroids.

\section{Acknowledgement}

The authors would like to thank prof. Jovan Miljković, MD, PhD, for the skin biopsy figure.
9. Ferkolj I, Hocevar A, Golouh R, Dolenc Voljc M. Infliximab for treatment of resistant pyoderma gangrenosum associated with Crohn's disease. Acta Dermatovenerologica Alpina, Pannonica, Adriat. 2006;15:173-7.

10. Ali M, Duerksen DR. Ulcerative colitis and Sweet's syndrome: a case report and review of the literature. Can J Gastroenterol. 2008;22:296-8.

11. Su WP, Liu HN. Diagnostic criteria for Sweet's syndrome. Cutis. 1986;37:167-74.

12. So JK, Carlos CA, Frucht CS, Cohen PR. Histiocytoid giant cellulitis-like Sweet's syndrome: case report and review of the literature. [Internet]. Vol. 21, Dermatology online journal. 2015 [cited 2018 Jan 21]. Available from: http://www.ncbi. nlm.nih.gov/pubmed/25780979.

13. Walker DC, Cohen PR. Trimethoprim-sulfamethoxazole-associated acute febrile neutrophilic dermatosis: case report and review of drug-induced Sweet's syndrome. J Am Acad Dermatol. 1996;34:918-23.

14. Fujii A, Mizutani Y, Hattori Y, Takahashi T, Ohnishi H, Yoshida S, et al. Sweet's syndrome successfully treated with granulocyte and monocyte adsorption apheresis. Case Rep Dermatol. 2017;9:13-8. 